\title{
Acerca de la interpretación en lingüística
}

Edith Le Bel

El problema de la interpretación en lingüística conlleva toda una serie de planteamientos previos sobre el objeto, el alcance y el método de esta ciencia en materia de interpretación.

En efecto, de un enfoque lingüístico a otro, cambian mucho estos parámetros.

Así, la perspectiva inmanentista del estructuralismo y del generativismo consolida la subordinación del sentido -del «sens»- a los signos.

«Ce qui compte, ce sont les lois du signe. D'elles seules dépend le sens» (Vignaux, 1981, pág. 94).

La lingüística de la enunciación y la pragmática lingüística aportan una alternativa a las limitaciones de las corrientes anteriores al resaltar el papel preponderante de la situación discursiva en la interpretación, aunque estos dos enfoques lingüísticos difieren en su campo de estudio y en su metodología.

\section{Niveles de interpretación en lingüística}

Saussure y los estructuralistas ven en la lengua, objeto de su análisis, un inventario de signos, un stock, a disposición del hablante. Consideran la lengua como «matriz» del habla y, por consiguiente, como «le dépôt sédimentaire des innombrables usages de la parole» (Seleskovitch, 1986, pág. 103). La «parole» «selon une métaphore qui a eu du succès (...) n'est rien d'autre que langue 'exécutée' par les individus, au sens même où une symphonie est exécutée par des musiciens» (Ducrot, 1989, pág. 159).

Chomsky reformula los conceptos de lengua y habla de Saussure con los de competencia y de actuación y pone al centro de su estudio, ya no la palabra en la lengua, sino la oración en la competencia, introduciendo la noción de «creatividad» del hablante, entendida como capacidad de generar una infinidad de oraciones a partir de un sistema reducido de reglas y no como la «creatividad libre y caprichosa de la parole» (Muñoz Romero, 1990). 
Por consiguiente, ambas lingüísticas sitúan su objeto de análisis fuera de las condiciones de producción de los enunciados.

Los locutores y receptores «ideales» de Chomsky no entran en consideración en su análisis: están puestos entre paréntesis.

La frase, en la estructura superficial, es por definición, ambigua pero «l'ambiguïté ne tient pas à la possible multiplicité des objets auxquels un texte peut se référer, mais à la multiplicité des indicateurs syntagmatiques (des structures) dont il est issu» (Dubois, 1969 pág. 101). La ambigüedad tampoco nace de alguna estrategia por parte de un locutor con una intención comunicativa determinada en una situación enunciativa dada.

Ahora bien, como dice Bourdieu (1975) criticando a Chomsky, «la langue est faite pour être parlée et il n'y a de discours que pour quelqu'un et dans une situation» (Pág. 23) o, dicho con las palabras de Seleskovitch (1986), «l'appréhension d'un vouloir dire à travers la langue et non la langue seule est à la base de l'interprétation »(Pág. 108). En este sentido, las lingüísticas enunciativas rehabilitan el papel de las circunstancias situacionales en la interpretación discursiva y, en particular, el protagonismo del YO enunciador en torno a quien se organizan los demás parámetros del acto enunciativo. Deja dề ser un sujeto ideal, universal en cuanto estas lingüísticas no le dan el mismo estatus que al receptor como en la teoría de la comunicación. En efecto, producción y reconocimiento interpretativo constituyen una disimetría en la relación locutor-receptor. Así, interpretar un signo, un enunciado o un texto presupone no ser «un simple récepteur-décodeur du message produit, mais un 'constructeur de signification'» (Charaudeau, 1978, pág. 74) en función del universo propio de cada «intérprete».

Por consiguiente, los enfoques enunciativos sitúan la semántica en el marco del intercambio verbal y de la disimetría producción/recepción.

Vemos que estamos muy lejos de la concepción saussuriana de significado en la lengua al dar prioridad al discurso como lugar de significación y de comunicación:

«Le sémiotique se caractèrise comme une propriété de la langue, le sémantique résulte d'une activité du locuteur qui met en action la langue» (Benveniste, 1974, pág. 64).

Así, sujeto y enunciación -esta «mise en action de la langue» pertenecen al discurso:

«YO y TU están incluidos en los modelos mismos, estableciéndose aś la existencia de un enunciador y de un destinatario en tanto que parámetros enunciativos» (Muñoz Romero, ibid).

Así, con los conceptos de enunciado y enunciación, nos alejamos también de la noción de frase. El enunciado se define como «token» o «ocurrencia» en oposición con la frase considerada como «type» (Cfr. Ducrot, 1984) y se define también como producto del acto de enunciación, como portador de sus huellas, dependiendo de sus parámetros para significar. Se constituye en objeto de estudio a partir del que se infieren los mecanismos de la enunciación. 
Por consiguiente, en estas perspectivas, la lengua es un sistema de signos interiorizado por los interlocutores, sistema que actualizan en la producción/recepción de enunciados singulares, lugar de significación y de interpretación.

A través de un acto singular de enunciación, «le sens naît de la perception d'une signification linguistique stable fusionnant avec la situation du moment». (Seleskovitch, 1986, pág. 112).

\section{Alcance del campo de la interpretación en lingüística}

Determinado el nivel de interpretación del signo -el discurso- a través de sus enunciados, lugar de inferencia de los mecanismos de la enunciación, y vista la necesidad de incorporar las condiciones de su producción que influencian el mecanismo de apropiación de la lengua por parte del locutor en función de una intención comunicativa, cabe preguntarse hasta dónde llega el campo de análisis del lingüista al servicio de la interpretación y cómo incorpora los parámetros situacionales sin salir de ese campo.

Dicho de otro modo: ¿Hasta qué punto el contexto enunciativo, el locutor y sus intenciones, así como el interlocutor y sus circunstancias, que por definición, pertenecen a lo extra-lingüístico, interesan al lingüista?

\section{Texto y contexto}

A la pregunta formulada, Coseriu (1962) da una primera respuesta:

«...el reconocimiento de los entomos es imprescindible para el análisis gramatical de los textos, pues los mismos esquemas formales pueden corresponder a funciones enteramente distintas en entornos distintos. En general, una lingüística propiamente funcional no puede descuidar los entornos, ni siquiera los «extraverbales», pues las funciones reales no se dan en la lengua abstracta, sino en el hablar concreto» (Pág. $320)$.

En efecto, cualquier cambio en la situación afecta el significado de un mismo enunciado. Los traductores e intérpretes lo saben. Un enunciado tan trivial como «On frappe», aislado de todo contexto, no se puede traducir. No se le puede asignar ningún valor enunciativo ni tampoco ilocutivo. Es imprescindible conocer la situación de enunciación, quién habla, dónde, cuándo, de qué o de quién, para qué y para quién, etc., para poder reconstruir el significado de un enunciado. Lederer (1986) insiste en este sentido:

«La perception d'une situation différente s'intégrant à un seul et même énoncé linguistique peut constituer tantôt une idée, tantôt une autre» (Pág. 44). 
Atlani (1984) también recalca la importancia de los parámetros situacionales en la traducción:

«Le choix entre différentes possibilités de traduction est souvent tributaire du contexte situationnel et/ou discursif dans lequel se présente l'énoncé» (Pág. 15).

Observamos que esta autora distingue, por consiguiente, dos tipos de contexto que pueden intervenir alternativa o cumulativamente en la interpretación.

La terminología utilizada para referirse a los parámetros extraverbales es fluctuante: «situación de comunicación», «contexto situacional» constituyen sus denominaciones más frecuentes. Se suelen distinguir de los parámetros verbales que, también, conocen una terminología cambiante, aunque menos variada. Se habla, en general, de «contexto lingüístico», de «contexto discursivo» o de «co-texto». La palabra «contexto», empleada sin adjetivación, suele abarcar ambos parámetros verbal y extraverbal.

La lingüística de la enunciación ha construido sus categorías a partir de la deixis al observar que los «embrayeurs» 0 «shifters» no cobraban su valor referencial sino a partir de la situación de enunciación:

«Il se trouve, en effet, que certaines classes d'éléments linguistiques présents dans l'énoncé ont pour rôle de «réfléchir» son énonciation, d'intégrer certains éléments du contexte énonciatif. Ces éléments sont partie intégrante du sens de l'énoncé et l'on ne peut ignorer ce à quoi ils seréfèrent si l'on entend comprendre ce sens». Maingueneau, 1981, pág. 7).

Esta lingüística ha extendido esta ley a todos los componentes del enunciado y ha ampliado también la deixis indexical a la deixis anafórica. Así, unas unidades lingüísticas están determinadas exclusivamente por el contexto discursivo, otras o bien por uno, o bien por otro.

En la interpretación de un enunciado, el contexto verbal puede ser necesario cuando no hay determinación extra-linguística. Por lo contrario, puede ser inútil y redundante cuando el contexto extraverbal aporta toda la información. Cuanto más rica esta información situacional, menos necesarios son los datos contextuales lingüísticos. Por consiguiente, contextos verbal y extraverbal tienen funciones similares:

"On est alors amené à poser que le contexte joue dans le discours le rôle de la situation» (Fuchs 1981, pág. 43).

Ahora bien, no se deben de confundir:

«Le contexte évoque des mots et la situation, des représentations» (Bally, 1965, pág. 44).

Pero ¿cuáles son estos parámetros contextuales y cómo se integran en el campo de análisis linguiístico? 
Para algunos lingüistas, se reducen al locutor, productor del acto enunciativo, al interlocutor susceptible de convertirse en locutor y al espacio-tiempo del acto enunciativo.

Otros lingüistas (Cfr. Bronckart, 1985) añaden a estos parámetros situacionales físicos (los interlocutores en un espacio físico dado) los parámetros sociales: el lugar social, las instancias sociales (enunciador-destinatario), la finalidad discursiva.

Esta distinción entre contexto enunciativo y contexto comunicativo (los parámetros de interacción social) está en la base del concepto de polifonía ${ }^{1}$.

La teoría de la polifonía enunciativa (Cfr. Ducrot 1984) distingue por una parte les personnages énonciatifs, sujetos soporte de la enunciación -el locuteur y el allocutairey, por otra parte, les personnages illocutoires, sujetos puestos en escena por la enunciación -el énonciateur y el destinataire -responsable y objeto, respectivamente, del acto ilocutivo:

«J'entends par locuteur un être qui, dans le sens même de l'énoncé, est présenté comme son responsable, c'est à dire comme quelqu'un à qui l'on doit imputer la responsabilité de cet 'énoncé» (Ducrot, 1984, pág. 193).

«J'appelle 'énonciateurs' ces êtres qui sont censés s'exprimer à travers l'énonciation, sans que pour autant on leur attribue de mots précis» (ibid., pág. 204).

«Je dirai que l'énonciateur est au locuteur ce que le personnage est à l'auteur» (ibid., pág. 205).

Desde un punto de vista distinto -el del traductor intérprete- Lederer (1986) distingue, por su parte la situación que define como «le cadre matériel..., tous les éléments formellement présents et perçus en même temps que le discours» (Pág. 44); el contexto verbal que define como una entidad paradigmática y psíquica, a saber «la présence simultanée d'un ensemble de mots dans la mémoire immédiate» y que esta autora compara con el «empan de l'appréhension visuelle» (ibid); el contexto cognitivo que es el «ensemble dynamique des informations qu'apporte à l'auditeur le déroulement du discours ou au lecteur celui de sa lecture» (ibid, pág. 45).

De hecho, Lederer establece estas distinciones contextuales para dar cuenta de los parámetros movilizados en la actividad interpretativa como proceso cognitivo:

«Il y a donc plusieurs étapes dans l'orientation du récepteur vers le sens réel du message: le contexte verbal, puis le contexte cognitif qui permet de dégager peu à peu un sens de l'énoncé, enfin le savoir et les connaissances ambiantes de l'auditeur/ lecteur sans lesquels le message risquerait de rester lettre morte» (ibid. pág. 47).

${ }^{1}$ El concepto de polifonía en lingüística fue desarrollado por Ducrot (Cfr. Ducrot et al. 1981 y Ducrot, 1984, cap. VIII) que hace «une extension (très libre) à la linguistique des recherches de Bakhtine sur la littérature» (1984, pág. 173) en contra del postulado, anclado en la tradición lingüística, según el cual «un énoncé isolé fait entendre une seule voix» (Ibid., pág. 171).

Siguiendo a Authier (1978) y a Plénat (1975), Ducrot critica, en particular, la perspectiva de Banfield (1979) sobre el estilo indirecto libre en la que, precisamente, esta autora no contempla la pluralidad de los sujetos, identificando al locutor con el «sujet de conscience». 
Si bien es verdad que estos parámetros inciden en la interpretación discursiva, quedan fuera del alcance del análisis lingüístico propiamente dicho, al referirse a aspectos tanto psicológicos como «culturales» -el «savoin» o las «connaissances ambiantes de l'auditeur/lecteur»-difíciles de «en casillan» teóricamente en una perspectiva lingüística.

En efecto, el contexto puede remitir a todo lo que nos rodea, a todos lo extralinguístico, a todas las determinaciones empíricas -psicológicas, sociológicas, antropológicas, etc. -que influyen en el acto enunciativo de cada locutor.

Ahora bien, entra en el campo de análisis del lingüista no solo porque constituye una condición imprescindible para acceder al significado textual y porque constituye el origen de la organización del discurso sino porque esta situación se encuentra «médiatisée par les moyens même de la langue» (Caron, 1978, pág. 499) que reorganiza la situación extralinguística en situación discursiva.

El locutor opera una selección en función de los recursos ofrecidos por la lengua y en función de su intención comunicativa.

$\mathrm{El}$ referente no es una entidad independiente, exterior al lenguaje que éste verbalizaría, sino una construcción lingüística. El discurso produce el referente:

«La 'signification' c'est alors la construction par le discours... d'un réseau de références auxquelles pourra renvoyer ce discours et qui permettront del' 'interpréter'. Le problème difficile de la référence peut ainsi trouver une solution si on l'envisage sous la forme de constructions de sens qui vont se faire grâce aux marques du langage et assurer relation entre éléments du linguistique et éléments extérieurs à ce linguistique (Vignaux, 1981, pág. 96).

Vemos, por consiguiente, que la lingüística enunciativa considera que texto y contexto mantienen una dependencia mutua.

Tal concepción devuelve al texto su autonomía lo que lleva a Vignaux a afirmar:

«N'importe quel discours une fois écrit ous retransmis, est détaché des caractéristiques de son apparition. Son sens n'est plus l'intention de celui qui l'a prononcé mais une intention qui peut, par d'autres, être identifiée, réidentifiée, et cette reconnaissance l'objectivise comme évènement de discourset non plus seulement comme évènement d'un sujet» (ibid, pág. 95).

A nuestro entender, es también definirlo como «évènement» del «lecteur/auditeun, volviendo a la noción de reconstrucción del significado textual reflejado por la disimetría en la relación de comunicación entre el acto de producción y de reconocimiento del texto y que confiere a este «lecteur-/auditeur» una relativa libertad de interpretación en función de sus propias circunstancias.

La pragmalingüistica, al igual que la lingüística enunciativa «stricto sensu» «entend, en droit, couvrir la totalité des données linguistiques» (Fuchs 1981 a, pág. 46).

La fuerza ilocutiva de un acto de habla, por ejemplo, se refleja en el orden de las palabras, en el acento lógico, en la entonación, en la puntuación, en el modo del verbo, en el tipo de frase, en la estructura léxica de los verbos, etc. 
Examinaremos, por consiguiente, las categorías formales y nocionales que ambas corrientes enunciativas movilizan al servicio de la interpretación desde sus respectivas metodologías.

\section{Categorías, nociones y enfoque metodológico de las corrientes enunciativas e interpretación}

«Les phénomènes linguistiques pris en charge par les théories de l'énonciation sont loin de se limiter aux embrayeurs» (Maingueneau, 1981, pág. 8).

En efecto, como comentábamos anteriormente, la lingüística enunciativa extendió su campo de estudio a la totalidad de las unidades enunciativas así como a las operaciones constitutivas del enunciado.

Pasó del estudio de las categorías deícticas de mostración a las de determinación, así como al léxico y a la sintaxis:

«Le concept de modalisation permet une analyse plus précise des moyens phrastiques utilisés pour traduire le procès d'énonciation» (Dubois, 1969, pág. 105).

Dubois subraya, en efecto, que en el estudio de las modalidades se distinguen:

- los modalizadores formalizados como los adverbios de «opinión»;

- las transformaciones modalizadoras como la énfasis o el pasivo facultativo;

- las interdependencias de niveles de registros de lengua -familiar, popular, literaria, etc.-

-las modalizaciones de la perspectiva del sujeto como la oposición «accompli/non accompli» de las formas verbales;

- las modalizaciones de los tipos de enunciados -directo, reproducido, etc.

La lingüística enunciativa pasó también del análisis de la modalidad al estudio de los distintos registros enunciativos como base de una tipología discursiva (Cfr. Benveniste, 1966 cap. XIX; Simonin-Grumbach, 1975, etc.), así como a los distintos modos de intervención del sujeto en el discurso.

Por consiguiente, se puede decir que la lingüística de la enunciación parte de marcas linguísticas y de categorías formales para desembocar en categorías nocionales como:

- La noción de distancia, «distance relative mise par le sujet entre lui-même et son énoncé» (Dubois, 1969, pág. 104);

- La noción de modalización, «l'adhésion que donne à son discours le sujet qui l'émet» (ibid., pág. 105).

- las nociones de transparencia y de opacidad es decir el grado de ambigüedad en función de la presencia mayor o menor del sujeto en el enunciado;

- la noción de tensión, la "prise en charge», el grado de implicación del sujeto en el enunciado. 
Ahora bien, todas estas nociones se inducen de sus anclajes textuales a partir de «repérages» formales.

El enunciado, por sus reglas, por la distribución de sus elementos constitutivos, traduce estas nociones.

Dubois (1969) plantea con todo el problema de saber si el flujo continuo del acto enunciativo se puede reducir a una serie de unidades discretas.

Antes de ir más adelante en el planteamiento de la operatividad epistemológica del análisis formal al servicio de la interpretación, veamos en qué la pragmática lingüística, esta «otra» lingüística enunciativa, difiere de la primera.

La noción de «prise en charge» o grado de implicación del sujeto en su enunciado en la que desembocaba la lingüística enunciativa «stricto sensu» está también contemplada, entre otras, por la pragmalinguística en su estudio de los actos de habla definidos a partir de la distinciones austinianas entre «constativo»y "performativo», «ilocutivo» y «perlocutivo» (Cfr. Austin 1971).

Searie (1969) considera los actos de habla como «las unidades fundamentales o mínimas de la comunicación lingüística» (Pág. 30). Se basa en una teoría del lenguaje vista como parte de una teoría general de la acción.

Ducrot y Todorov (1972) afirman en este sentido:

«En tantqu'action particulière,l'énonciationrelève d'une étude totale du comportement dans la société et plus particulièrement de l'anthropologie linguistique» (pág. 407).

Ducrot (1984) confirma esta acepción de la pragmalingüística:

«Si l'on donne pour objet à la pragmatique l'action humaine en général, le terme de pragmatique du langage peut servir à désigner, dans cet ensemble de recherches celles qui concernent l'action humaine accomplie au moyen du langage» (pág. 173).

En la misma línea, Verschueren (1980) opina que la pragmática «est analogue à cette branche de la sociologie qu'on appelle ethno-méthodologie (...) et qui a pour objet le monde des croyances qui est au fondement du comportement social» (Pág. 283).

Se considera el acto de habla bajo tres puntos de vista:

- como acto proposicional o locutivo: el acto mismo de decir;

- como acto ilocutivo: lo que se hace hablando promesa, amenaza, deseo, aserción, etc.;

- como acto perlocutivo: lo que se produce por el hecho de hablar, los efectos sobre los sentimientos, las ideas o acciones del oyente (Cfr. Austin 1971).

La pragmalingüística estudia también los mecanismos conversacionales, estableciendo en la línea de los trabajos de Grice (1975), Gordon-Lakoff (1973) o Anscombre (1980), las reglas, implicaturas y postulados constitutivos del intercambio verbal, distinguiendo los niveles de actuación de los actos de habla (primitivos, derivados, marcados, manifiestos o no). 
El estudio de las presuposiciones es también objeto del análisis pragmalingüístico (Cfr. Ducrot 1972 y 1978). Schmidt (1973) las define como «los supuestos de comunicación que afirma un hablante con un enunciado, indiferentemente de si el enunciado es positivo o negativo» (citado en Lewandowski, 1981, pág. 272).

Un enunciado, al conllevar una presuposición $u$ otra, introduce una cierta indefinición en las relaciones intersubjetivas en el discurso. Esto lleva a Ducrot (Cfr. Ducrot-Todorov, 1972) a decir:

«Présupposer serait alors un acte de parole ayant une valeur illocutoire au même titre que promettre, ordonner, interroger» (Pág. 347-348).

Como subraya Fuchs (1981), vemos que la corriente pragmalingüística «manifeste clairement une tendance à privilégier 'les modes du dire 'sur le 'dit', la dimension de l'implicite, du sous-entendu, du dérivé, du présupposé, de l'allusif, du montré, sur celle de l'explicite, de l'asserté, du dit, du posé; l'étude des images de l'énonciation sur celle du contenu de l'énoncé» (Pág. 46).

Por esta razón, Recanati (1980) intenta rehabilitar la noción de «locutivo» que Grice (1968 y 1969) o Ducrot (1972 o 1984) tienden a menospreciar, privilegiado «l'image de l'énonciation».

A pesar de sus divergencias, nos parece, con todo, que ambas corrientes enunciativas coinciden en la meta de cubrir el conjunto del campo lingüístico y en integrar en su análisis el parámetro «sujeto»" aunque no se sabe bien todavía qué estatus teórico atribuirle: «...méfiance à l'égard des opérations mentales auxquelles on n'a pas accès, essais pour jeter des ponts (prudents) entre linguistique et psycholinguistique (Culioli) ou au contraire rejet radical de tout lien entre opérations linguistiques et opérations du langage (Ducrot)» (Fuchs, 1981 a, pág. 51).

\section{Para concluir:}

Hemos visto que las corrientes enunciativas han marcado un giro metodológico importante al situar el lugar de significación lingüística en el nivel discursivo y al establecer una distinción fundamental entre «frase» y «enunciado». Este último está concebido como resultado de un acto comunicativo singulary comolugar de inscripción de las operaciones linguísticas movilizadas para su constitución. Tal distinción coloca el acto interpretativo a un nivel de actividad de reconstitución del significado -y no de simple «décodage»- en función de los «repérages» textuales del acto enunciativo.

Hemos visto, también, que no queda resuelto de momento el estatus teórico de la articulación entre sujeto lingüístico y sujeto extralingüístico es decir entre el campo de análisis lingüístico al servicio de la interpretación-las marcas formales de la inscripción

2 «Ce sujet d'énonciation est en effet à la source à la fois de la référence et de la modalisation* (Maingueneau, 1986, pág. 81). 
del sujeto en el enunciado- y los parámetros situacionales de tipo sociológico, psicológico o cultural que condicionan sus actos de habla.

«... autour du locuteur on a aujourd'hui soit le discours pseudo-grammatical..., soit les discours qui font surgir les grandes forces de l'intention ou de la conscience. Dans le premier cas, nous ne sommes pas certain qu'il s'agisse d'une réflexion sur la production des actes de parole, dans le second cas, nous craignons qu'il manque une théorie». (Grunig, 1979, pág. 31).

Sin querer entrar en este delicado terreno epistemológico, nos parece importante plantear, a la luz de esta problemática, la necesidad y los límites del análisis formal al servicio de la interpretación. Nos parece imprescindible establecer la distinción entre los diferentes sujetos lingüísticos -enunciativos e ilocutivos- y los sujetos de carne y hueso de la realidad empírica extralingüística. Sin embargo, no debemos olvidar que interpretamos un texto desde y dentro de esta realidad empírica. Por consiguiente, si uno puede justificar una interpretación contextual a partir de «repérages» formales en el texto, esta interpretación no es mero producto de una actividad de restitución del contexto a partir del texto. En efecto, el trabajo de reconstrucción del significado se hace también en función de otros múltiples parámetros, saberes extralingüísticos -psicosocioculturales- que la condicionan.

Por esto, pensamos que el papel -importantísimo- de la lingüística en materia de interpretación se limita a una justificación formal de una lectura textual informada, entre otros, por los parámetros enunciativos inscritos en este texto.

\section{Referencias bibliográficas}

ANSCOMBRE, J.C. (1980): Voulez-vous dériver avec moi? Communications, 30, 61-124.

ATLANI, F. (1984): «On» l'illusionniste, in Grésillon, A. et al.: La langue au ras du texte. Paris, Presses Universitaires.

AUSTIN, J. L. (1970), TRAD. FR.) Quand dire c'est faire. Paris, Seuil.

AUTHIER, J. (1978): Les formes du discours rapporté, D.R.L.A.V., 17, 1-88.

BALLY, C. (1965) Linguistique générale et linguistique française, Beme, $4^{\mathrm{a}} \mathrm{ed}$.

BANFIELD, A. (1979): Où l'épistémologie, le style, la grammaire rencontrent la théorie littéraire, Langue Française, 44, 9-26.

BENVENISTE, E. (1966 y 1974) Problèmes de Linguistique Générale, T. I y II. Paris, Gallimard.

BOURDIEU, P. et al. (1975): Le fétichisme de la langue et l'illusion du communisme linguistique. Actes de la recherche en sciences sociales, 4, 2-32.

BRONCKART,J.P.(1985): Lefonctionnement des discours. Paris, Delachaux et Niestlé. CARON, J. (1978): Pragmatique du langage et logique naturelle: Recherches psycholinguistiques sur quelques connecteurs du discours. Thèse pour le Doctorat d’Etat. Université de Bordeaux II. 
CHARAUDEAU, P. (1978): Les conditions linguistiques d'une analyse du discours. Thèse. Paris IV, 1977. Publications de l'Université de Lille III.

COSERIU, E. (1962): Teoría del lenguaje y lingüistica general. Paris, Gredos.

DUBOIS, J. (1969): Enoncé et énonciation. Langages, 13, 100-110.

DUCROT, O. (1984): Le dire et le dit. Paris, Ed. de Minuit.

DUCROT, O. (1989): Logique, structure, énonciation. Lectures sur le langage. Paris, Ed. de Minuit.

DUCROT, O.; TODOROV, T. (1972): Dictionnaire encyclopédique des sciences du langage. Paris, Seuil.

DUCROT, O, et al. (1980): Les mots du discours. Paris, Ed. de Minuit.

FUCHS, C. (1981): Les problématiques énonciatives. Esquisse d'une présentation historique et critique. D.R.L.A.V. 25, 35-60.

GORDON,D; LAKOFF, G. (1971): Conversational postulates. C.L.S., 7 (Trad. fr. 1973: Postulats de conversation. Langages, 30, 32-56.

GRICE H, P. (1968): Utterer's meaning sentence meaning and word meaning. Foundations of Language, 4, 225-242.

GRICE, H.P. (1975): Logic and conversation, in COLE, MORGAN, eds.: Syntax and Semantics 3: speech acts. New York, Academic Press, 41-48 (Trad. fr. 1979: Communications, 30, 57-72.

GRUNIG, B.N. (1979): Pièges et illusions de la pragmatique linguistique. Modèles linguistiques, 2, 7-38.

LEWANDOWSKI, T. (1981): Diccionario de lingüística. Madrid, Cátedra.

MAINGUENEAU,D.(1981): Approche del'énonciation en linguistiquefrançaise.Paris, Hachette.

MAINGUENEAU, D. (1986): Eléments de linguistique pour le texte littéraire. Paris, Hachette.

MUÑOZ ROMERO, M. (1990): La noción de situación en lingüística. Actas del Iler Simposio Internacional de la Asociación Andaluza de Semiótica. Universidad de Granada.

PLENAT, M. (1979): Sur la grammaire du style indirect libre. Cahiers de grammaire, 1, 95-137. Université de Toulouse-Le Mirail.

RECANATI, F. (1980): Qu'est-ce qu'un acte locutionnaire? Communications, 32, 190-239.

SEARLE, J.R.: Speech-acts: An essay on the philosophy of language. Cambridge University Press. (Trad. fr. 1972: Les actes de langage. Paris, Hermann).

SELESKOVITCH, M.; LEDERER, M. (1986): Interpréter pour traduire. Paris, Didier Erudition.

SIMONIN, J. (1975): Pour une typologie des discours, Langue, discours, société. Pour Emile Benveniste. Paris, Seuil, 85-121.

VERSCHUEREN, J.(1980): A la recherche d'une pragmatique unifiée. Communications, $32,274-284$.

VIGNAUX, G. (1981): Enoncer, argumenter: opérations du discours, logiques du discours. Langue Française, 50, 91-116. 